\title{
Isolation and phenotypic characterization of cancer stem-like side population cells in colon cancer
}

\author{
LONG FENG, JIAN-BING WU and FENG-MING YI \\ Department of Oncology, The Second Affiliated Hospital of Nanchang University, Nanchang, Jiangxi 330006, P.R. China
}

Received May 27, 2014; Accepted February 26, 2015

DOI: $10.3892 / \mathrm{mmr} .2015 .3801$

\begin{abstract}
Previous studies in cancer biology suggest that chemotherapeutic drug resistance and tumor relapse are driven by cells within a tumor termed 'cancer stem cells'. In the present study, a Hoechst 33342 dye exclusion technique was used to identify cancer stem-like side population (SP) cells in colon carcinoma, which accounted for $3.4 \%$ of the total cell population. Following treatment with verapamil, the population of SP cells was reduced to $0.6 \%$. In addition, the sorted SP cells exhibited marked multidrug resistance and enhanced cell survival rates compared with non-SP cells. The SP cells were able to generate more tumor spheres and were CD133 positive. Subsequent biochemical analysis revealed that the levels of the adenosine triphosphate-binding cassette sub-family G member 2 transporter protein, B-cell lymphoma anti-apoptotic factor and autocrine production of interleukin-4 were significantly enhanced in the colon cancer SP cells, which contributed to drug resistance, protection of the cells from apoptosis and tumor recurrence. Therefore, the findings suggested that treatment failure and colon tumorigenesis is dictated by a small population of SP cells, which indicate a potential target in future therapies.
\end{abstract}

\section{Introduction}

Colorectal cancer is the third most common type of cancer and remains one of the leading causes of cancer-associated mortality worldwide (1). Despite advances in the treatment of cancer, treatment failure and tumor recurrence remain a problem. Previously, it was suggested that all neoplastic cells within a tumor are potentially tumorigenic. However, according to subsequent studies, a small subset of cancer initiating cells, termed cancer stem cells (CSCs) have been suggested as the factor involved in drug resistance and

Correspondence to: Dr Long Feng, Department of Oncology, The Second Affiliated Hospital of Nanchang University, 1 Minde Road, Nanchang, Jiangxi 330006, P.R. China

E-mail: fenglong491@gmail.com

Key words: cancer stem cells, chemotherapy, side population cells, tumor recurrence, apoptosis tumorigenesis $(2,3)$. The presence of CSCs, their stem-like role and their implications in the treatment of cancer have been well established in several types of cancer (4-8). Previous studies have reported that there are two different methods to isolate CSCs, based on the expression of CD133 and the Hoechst 33342 dye exclusion technique. In the latter, a small population of cancer cells can be been identified at the side of the overal population in fluorescence-activated cell sorting (FACS) analysis, which exclude Hoechst 33342 dye and are, therefore, termed side population (SP) cells. These SP cells share the characteristic features of CSCs, including the expression of stem cells surface markers, high level of tumorigenicity and differentiation potential (9-12). Furthermore, the SP cells have high expression levels of adenosine triphosphate-binding cassette (ABC) transporters, enhanced DNA-repair capacity and resistance to apoptosis, which leads to chemoresistance and tumor relapse (13). Therefore, the isolation and characterization of SP cells can assist in identifying and targeting CSCs and, as a result, increase long-term disease-free survival rates.

It was previously reported, based on the expression of CD133, that isolated colon CSCs are highly resistant to apoptosis, associated with the autocrine production of interleukin (IL)-4 (14). Previous studies on several types of cancer have demonstrated that IL-4 is involved in resistance to apoptosis and increased expression of antiapoptotic proteins (15). Therefore, the present study was designed to identify colon CSCs, based on the Hoecst 33342 dye exclusion technique. Furthermore, the sorted colon CSCs were analyzed for CD133 positivity, drug resistance and the expression of IL-4.

\section{Materials and methods}

Tissue collection and cell culture. Colon adenocarcinoma tissue samples were collected from patients during colon resection $(\mathrm{n}=10,20-60$ years old, 5 male and 5 female), according to standard ethical standards. The study was approved by the ethics committee of The Second Affiliated Hospital of Nanchang University (Nanchang, China) and consent was obtained from all patients. The diagnosis of colon cancer was made based on clinical, pathological and laboratory tests performed by qualified physicians. Following isolation, the tissues were diagnosed, based on their histological type and grade. The cancer tissues were washed thoroughly in phosphate-buffered saline (PBS; Sigma-Aldrich, St. Louis, MO, USA) solution, containing antibiotics and were incubated overnight in Dulbecco's modified 
Eagle's medium (DMEM)/F12 (Gibco Life Technologies, Carslbad, CA, USA) containing penicillin $(500 \mathrm{U} / \mathrm{ml})$, streptomycin $(500 \mathrm{~g} / \mathrm{ml})$, and amphotericin B $(1.25 \mu \mathrm{g} / \mathrm{ml})$ (Gibco Life Technologies) at room temperature. Enzymatic digestion was performed using collagenase $(1.5 \mathrm{mg} / \mathrm{ml})$ (Gibco Life Technologies) and hyaluronidase $(20 \mu \mathrm{g} / \mathrm{ml}$; Sigma-Aldrich $)$ in PBS for $1 \mathrm{hr}$ at room temperature. The cells were cultured in DMEM with $10 \%$ fetal bovine serum (FBS; Sigma-Aldrich), supplemented with antibiotics, and maintained in T-75 flasks at $37^{\circ} \mathrm{C}$ in a humidified $5 \% \mathrm{CO}_{2}$ and $95 \%$ air atmosphere. At $90 \%$ confluence, the cells were removed from the culture flask using trypsin-EDTA (0.25\%: 53 mM EDTA; Sigma-Aldrich) washed in distilled water, and the cells were suspended in $10 \%$ DMEM. The number of cells were counted using a hemocytometer (Z359629; Bright-Line; Sigma-Aldrich).

FACS analysis. The following experimental groups were included in the present study: The control group, containing cells + Hoechst 33342 dye $(n=5)$; and the drug-treated group, containing cells + verapamil (Sigma-Aldrich) + Hoechst 33342 dye $(n=5)$. The techniques for the Hoechst 33342 dye labeling and immunofluorescence were obtained from Dr Wanshan $\mathrm{Li}$ of the Department of Oral and Maxillofacial Surgery, Chongqing Medical University (Chongqing, China). The cells $\left(\sim 10^{6}\right.$ cells $/ \mathrm{ml}$ of $10 \%$ DMEM $)$ were labeled with Hoechst 33342 stock (Sigma-Aldrich)-bis-benzimide $(5 \mu \mathrm{l} / \mathrm{ml})$, with either the dye alone, or in combination with verapamil $(0.8 \mu 1 / \mathrm{ml})$. The cells were resuspended in $500 \mu 1$ Hank's balanced salt solution (HBBS; Sigma-Aldrich), containing 10 mM HEPES for FACS analysis. Finally, the cells were counterstained with $2 \mu \mathrm{g} / \mathrm{ml}$ propidium iodide (PI; Sigma-Aldrich). The cells were sorted into SP and non-SP cells using a flow cytometer (Attune NxT; Life Technologies, Grand Island, NY, USA), and the sorted cells were then cultured and maintained in DMEM/F-12, supplemented with $10 \%$ FBS at room temperature.

Immunocytochemistry. The sorted SP cells and non-SP cells were seeded in $35 \mathrm{~mm}$ culture plates $(\sim 100 \mu \mathrm{l})$ with $1 \mathrm{ml} 10 \%$ DMEM. Following incubation overnight, the cells were rinsed with PBS and fixed in 4\% paraformaldehyde (Sigma-Aldrich). Subsequently, the cells were blocked with $1 \%$ bovine serum albumin (BSA; Sigma-Aldrich)-Tris-buffered saline (TBS; Sigma-Aldrich) with RNase (10 $\mu 1 / 1,000 \mu 1$ 3\% BSA-TBS). After $1 \mathrm{~h}$ incubation at room temperature, the cells were rinsed with PBS and were incubated with primary antibody against CD133 (cat no. orb99113; polyclonal, rabbit; Biorbyt, San Francisco, CA, USA) in 1\% BSA-TBS (1:100; $2 \mu \mathrm{l} / 200 \mu \mathrm{l}$ ), incubated overnight at $40^{\circ} \mathrm{C}$. The cells were then washed with $1 \mathrm{X}$ PBS, followed by incubation with fluorescein isothiocyanate (FITC)-conjugated secondary antibody (1:100 in $1 \%$ BSA-TBS; goat anti-rabbit immunoglobulin G with alkaline phosphatase markers; cat. no. sc-2043; Santa Cruz Biotechnology, Inc., Dallas, TX, USA), at room temperature for $1 \mathrm{~h}$. The cells were then washed again with PBS, and PI was added ( $1 \mu 1 / 200 \mu \mathrm{l}$ PBS). The tumor spheres formed were counterstained with Hoechst 33342 staining (200 $\mu \mathrm{l}$ for $15 \mathrm{~min}$; Life Technologies) to visualize the nuclei. The cells were then viewed under a confocal laser scanning microscope (Leica TCS; Leica Microsystems, Inc., Buffalo Grove, IL, USA). Image analysis and figures were prepared using Adobe Photoshop CS6 (Adobe Systems, Inc., San Jose, CA, USA).

Cell resistance assay. The SP and non-SP cells were cultured in 96 -well plates at a concentration of $1 \times 10^{3}$ cells/plate at $37^{\circ} \mathrm{C}$. After 24 h, 5-fluorouracil (5-FU; Sigma-Aldrich) was added to the cultures to a final concentration of $10 \mu \mathrm{g} / \mathrm{ml}$. The cells were also treated with cisplatin ( $20 \mu \mathrm{mol} / \mathrm{l}$; Sigma-Aldrich), paclitaxel ( $2 \mu \mathrm{mol} / 1$; Sigma-Aldrich) and oxaliplatin $(100 \mathrm{mM}$; Sigma-Aldrich). The plates were placed in a hatch box for $48 \mathrm{~h}$, following which, each well was supplemented with $10 \mu \mathrm{l}$ Cell Counting kit-8 (CCK-8) solution, and the plates were incubated for $3 \mathrm{~h}$ at $37^{\circ} \mathrm{C}$. The mean optical density (OD) at $450 \mathrm{~nm}$ was determined using a UV/Vis spectrophotometer (UV-5800; Metash, Shanghai, China), and was represented as a graph. The resistance of the cells in the two groups was calculated using the following formula: Cell resistance rate $(\%)=($ experimental group OD450 / control group OD450) x 100, as described previously (16). Furthermore, the analysis of cell death of colon cancer spheres was evaluated using orange acridine/ethidium bromide staining (200 $\mu \mathrm{l}$ for $15 \mathrm{~min}$; Sigma-Aldrich), which was viewed under confocal microscopy (LSM 510; Carl Zeiss $\mathrm{GmbH}$, Jena, Germany). Images were captured and processed using Adobe Photoshop CS6 (Adobe Systems, Inc.).

Sphere formation assay. The sorted SP cells and non-SP cells were plated at a density of 1,000 cells $/ \mathrm{ml}$, resuspended in tumor sphere medium, containing a serum-free 1:1 mixture of Ham's F-12/DMEM (Sigma-Aldrich), N2 supplement, 10 $\mathrm{ng} / \mathrm{ml}$ human recombinant $\mathrm{bFGF}$ and $10 \mathrm{ng} / \mathrm{ml}$ epidermal growth factor (Sigma-Aldrich). The cells were then cultured in ultra-low attachment plates for $\sim 2$ weeks ay $37^{\circ} \mathrm{C}$. The sorted SP and non-SP cells were seeded at a low density of 20 cells/L, and the number of generated spheres, measuring $>100 \mathrm{~lm}$, were counted following 7 days of culture.

Biochemistry. The expression levels of proteins were analyzed using western blot analysis. The proteins were extracted from the SP and non-SP cells. Cell pellets were resuspended in ice-cold NP-40 lysis buffer (50 mM Tris-HCl, pH 7.5, $150 \mathrm{mM}$ $\mathrm{NaCl}, 1$ mM EGTA, 1\% NP-40) containing protease inhibitors., and the protein concentrations were determined using a Bradford assay (Bio-Rad Laboratories, Inc., Hercules, CA, USA). Following $10 \%$ sodium dodecyl sulfate-polyacrylamide gel electrophoresis and transfer onto nitrocellulose membranes (Bio-Rad Laboratories, Inc.), the gels were incubated with the following primary antibodies: Rabbit anti-human polyclonal ABC sub-family G member 2 (ABCG2; 1:500; cat. no. AV43649; Sigma-Aldrich), rabbit polyclonal Bcl-2 (1:500; cat. no. sc-492, Santa Cruz Biotechnology, Inc.) and rabbit polyclonal IL-4 (1:500, cat. no. sc-7919, Santa Cruz Biotechnology, Inc.), secondary antibody (goat anti-rabbit immunoglobulin $\mathrm{G}$ with alkaline phosphatase markers; 1:1,000, cat. no. sc-2043; Santa Cruz Biotechnology, Inc.) and chemiluminescence reagent. The blots were detected and scanned using a densitometer (Bio-Rad GS-710; Bio-Rad Laboratories, Inc.).

Statistical analysis. One-way analysis of variance and Student's t-test were performed to determine significant 

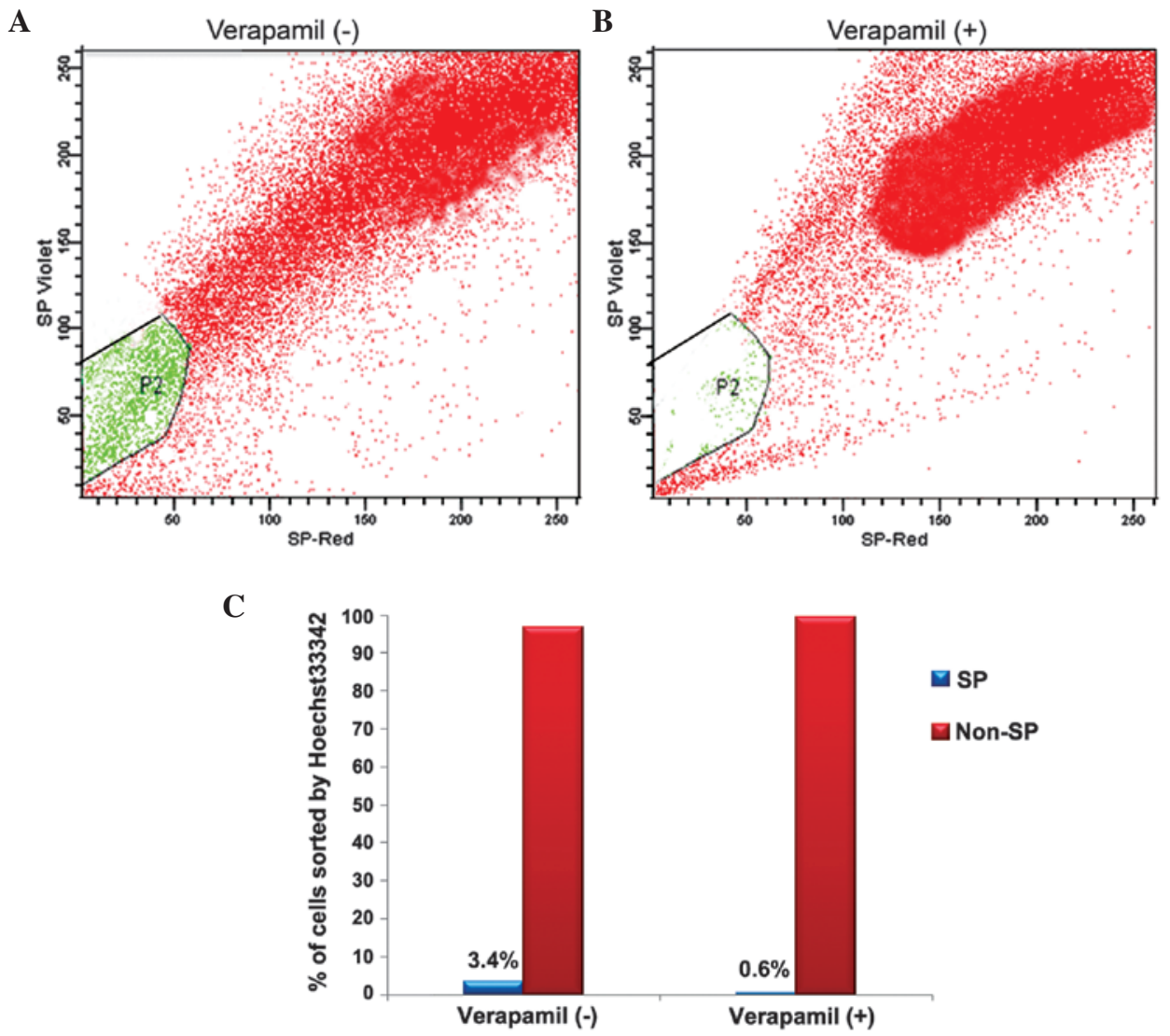

Figure 1. (A) Representative image of the dot plot analysis of FACS, indicating the presence of SP cells (P2-gated region). (B) Dot plot analysis revealing a reduction in the SP cell population upon treatment with verapamil. (C) Quantitative graph constructed from the FACS dot blot analysis. FACS, fluorescence-activated cell sorting; SP, side population.

A

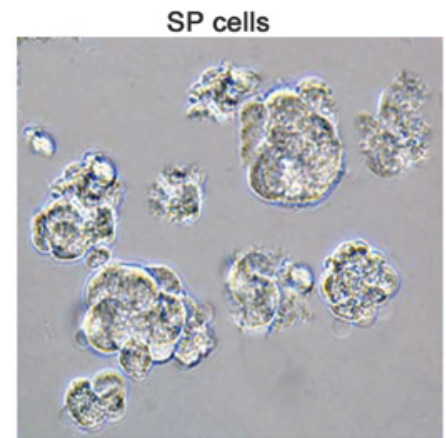

B

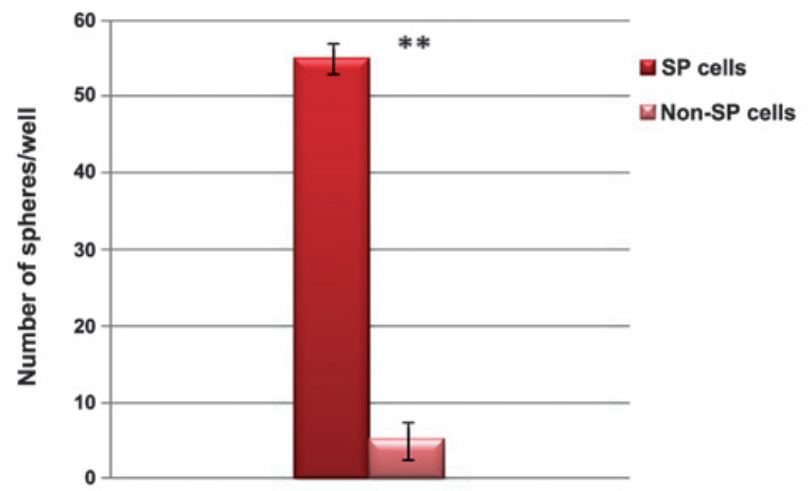

Non-SP cells

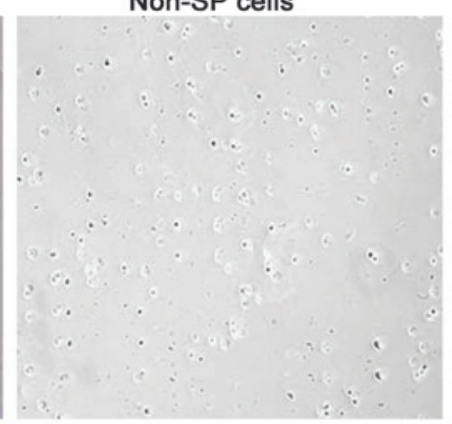

C

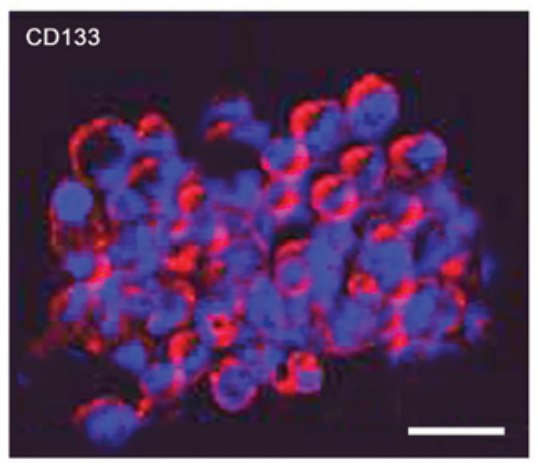

Figure 2. (A) Phase contrast microscopic images of tumor spheres generated by colon cancer SP cells. (B) Quantification of the number of spheres generated by the SP and non-SP cells, which were counted following 7 days of culture. The SP cells produced significantly more tumor spheres compared with the non-SP cells. ${ }^{* *} \mathrm{P}<0.05$, compared with non-SP cells. (C) Confocal microscopic analysis of the SP cell-generated tumor spheres, which exhibited positivity to CD133 (Red staining). The nuclei were counterstained using Hoechst 33342 (blue staining). Scale bar=20 $\mu \mathrm{m}$. SP, side population. 
A

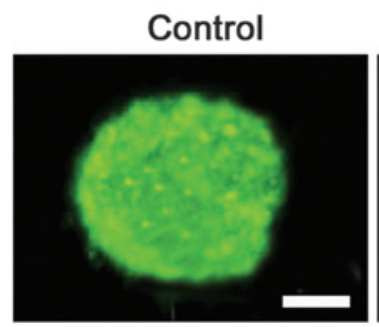

Oxaliplatin

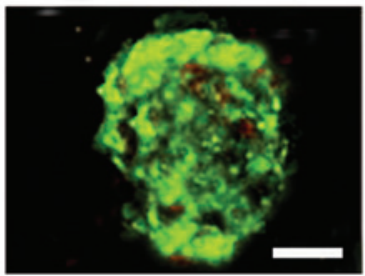

$5-\mathrm{FU}$

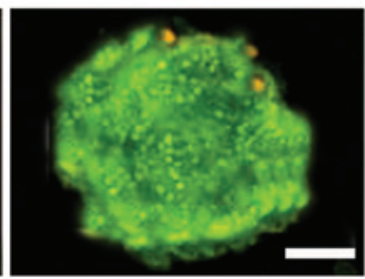

B

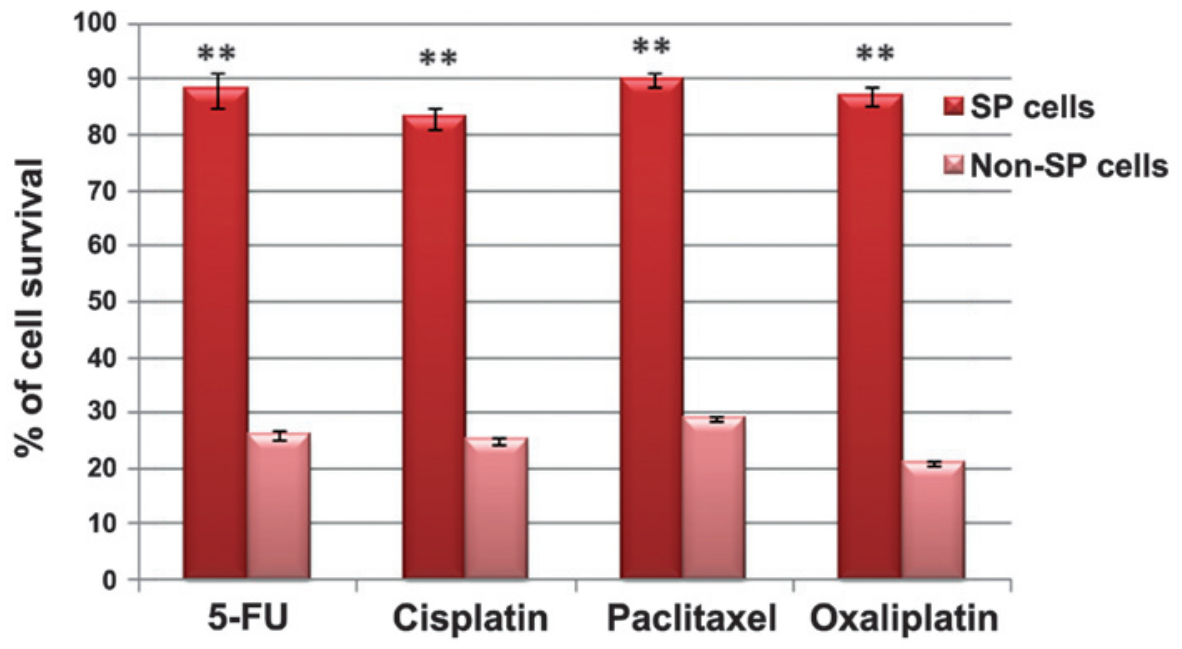

Figure 3. (A) Immunofluorescence analysis of the tumor spheres generated by colon cancer SP cells. The spheres were treated with 5-FU and oxaliplatin, and subsequently stained with orange acridine/ethidium bromide to visualize the cells, which had undergone cell death. Scale bar=20 $\mu \mathrm{m}$. (B) Comparison of the cell survival rates of the SP cells and non-SP cells following treatment with 5-FU, cisplatin, paclitaxel and oxaliplatin. ${ }^{* *} \mathrm{P}<0.05$, compared with non-SP cells. $\mathrm{SP}$, side population; 5-FU, 5-fluorouracil.

differences between the treatment and control groups using SAS statistical software (SAS Institute Inc., Cary, NC, USA). $\mathrm{P}<0.05$ was considered to indicate a statistically significant difference.

\section{Results}

FACS analysis of SP cells containing multidrug resistance transporter 1 (MDR1) using Hoechst 33342. The present study analyzed the colon cancer cells for the presence of cancer stem-like SP cells using a Hoechst 33342 dye exclusion method. During FACS analysis, the live cells were selected using PI staining, which was excluded by the dead cells (P1-gated region of Fig. 1A and B). The FACS analysis identified $\sim 3.4 \%$ of the SP cells from the colon cancer, which expelled Hoechst 33342 dye and occurred as a distinct population (P2 region of Fig. 1A). Hoechst 33342 efflux by SP cells is an active process by MDR1, an ABC transporter transmembrane protein. Therefore, the cells were subsequently treated with verapamil, an MDR1 inhibitor, which inhibits drug efflux by the cells. Following treatment with verapamil, the presence of SP cells was reduced between $3.4 \%$ and $0.6 \%$ (P2-gated region of Fig. 1B). Therefore, these data suggested that the presence of SP cells and the expression of $\mathrm{ABC}$ transporters in colon cancer may be responsible for chemotherapeutic drug efflux and subsequent chemoresistance.

Characterization of tumor spheres formed by colon cancer SP cells. The present study subsequently aimed to characterize the

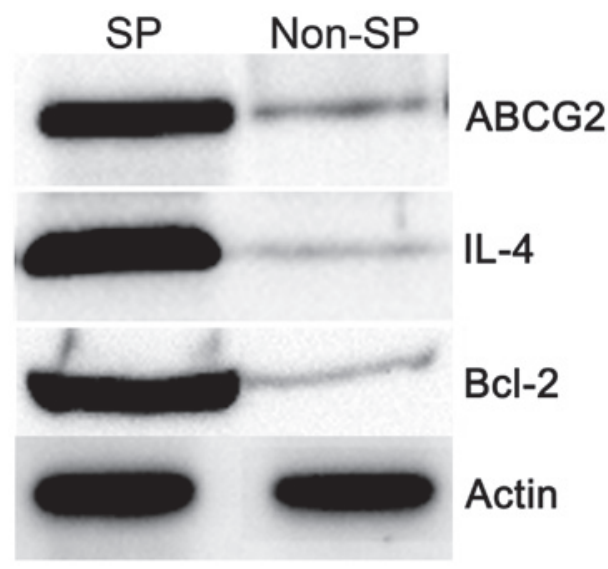

Figure 4. Western blot analysis of the protein expression levels in SP and non-SP cells. Equal concentrations of protein were loaded per lane. Actin was used as a loading control. SP, side-population; ABCG2, adenosine triphosphate-binding cassette sub-family G member 2; IL, interleukin; Bcl, B-cell lymphoma.

FACS-sorted SP cells by analyzing the ability of the SP cells to form tumor spheres and by determining their positivity to CD133 under standard adherent conditions. The sphere formation assay revealed that the SP cells readily formed tumor spheres and formed a cluster of sphere-like cells compared with the non-SP cells (Fig. 2A). These SP cells exhibited faster growth rates and spheres were observed on day 5 , unlike in the non-SP cells. The total numbers of tumor spheres formed by the SP and non-SP cells following 7 days of culture were also 
examined. As shown in Fig. 2B, the number of tumor spheres formed by the SP cells in serum-free medium was significantly higher than the number formed by the non-SP cells. Subsequently, the tumor spheres were analyzed for CD133 positivity by immunofluorescence. The tumor spheres generated by the SP cells exhibited positive expression of CD133 (Fig. 2C). These findings indicated that the cancer stem-like $\mathrm{SP}$ cells were markedly tumorigenic.

Multidrug resistance and resistance to apoptosis in colon cancer SP cells. For characterization, the sorted colon cancer SP and non-SP cells were subjected to a drug-resistance assay in order to determine the rate of cell survival following treatment with several chemotherapeutic drugs. Immunofluorescence analysis revealed that the tumor spheres contained more viable cells upon treatment with drugs, including 5-FU and oxaliplatin (Fig. 3A). The percentage of cell survival was also quantified for the SP and non-SP cells following treatment with cisplatin, paclitaxe, 5-FU and oxaliplatin. Following treatment with these drugs, the SP cells exhibited significantly higher survival rates compared with the non-SP cells (Fig. 3B). Furthermore, the biochemical data revealed that the SP cells exhibited elevated expression levels of the ABCG2 MDR1 transporter protein, the antiapoptotic factor, $\mathrm{Bcl}-2$, and autocrine secretion of IL-4, whereas the levels of these proteins were comparatively lower in the non-SP cells (Fig. 4). These results suggested that the colon cancer SP cells were highly multidrug resistant with a reduced rate of apoptosis, which contributed to enhanced survival following treatment with multiple drugs.

\section{Discussion}

Cancer is a disease, which contains heterogenous populations of cells exhibiting multiple differentiation due to randomly occurring successive mutations. It has been suggested that cancerous growth, metastasis and tumor recurrence are orchestrated by a small population of cells, termed CSCs (17). According to the CSC theory, the traditional therapeutic approaches, used to eradicate the majority of tumor cells and induce repression of tumor lesions, leave the CSCs unaffected, which is responsible for treatment failure and tumor recurrence. Thus traditional treatment fails to prevent disease relapse and tumor metastasis (3). Therefore, it is essential to develop a novel therapeutic drug, which can efficiently target the CSCs and CSC-mediated pathways. However, these hypotheses remain to be confirmed. Several reports have demonstrated that CSCs can be isolated and characterized based on either the use of a Hoechst 33342 dye exclusion technique or cell surface markers, including CD133 (7). Cells, which exclude Hoechst 33342 dye are referred to as SP cells, and share characteristic features of CSCs, including tumor initiating capacity, expression of stem-like genes and resistance to chemotherapeutic drugs (18). The results of the present study demonstrated that $3.4 \%$ of the cells in colon cancer were cancer stem-like SP cells, and their prevalence is reduced upon treatment with verapamil, an MDR1 inhibitor.

It was previously reported that, in colon cancer, a small population of CD133 positive cells are able to form spheres and are highly tumorigenic (19). Similar observations have been made for other types of solid tumor, including those in melanoma, and brain, ovarian, prostate and breast carcinoma (4-8). Furthermore, Dean et al reported that CSCs have the ability to resist death-inducing signals, as well as enhance the expression level of antiapoptotic proteins and high levels of drug transporters $(13,20)$. In accordance, the present study demonstrated high expression levels of $\mathrm{ABC}$ transporter proteins and the Bcl-2 anti-apoptotic factor in SP cells by western blot analysis. In addition, the tumor spheres generated by the SP cells were CD133 positive and effectively resisted chemotherapeutic drugs, including 5-FU, cisplatin, oxaliplatin and paclitaxel. This confirmed that CSCs exhibit chemotherapy resistance and are highly tumorigenic.

Considerable data has suggested that the secretion of certain interleukins, including IL-3 and IL-4, promotes and increases the survival of cancer cells by upregulating anti-apoptotic factors (21-23). In addition, neutralization of these IL secretions effectively increase the number of death receptors (14). The present study demonstrated that colon cancer SP cells also exhibited increased expression levels of IL-4, together with upregulated protein levels of Bcl-2. These results confirmed that IL-4-dependent protection is involved in cell death resistance in colon CSCs. Similar to these findings, it has been previously demonstrated that the secretion of IL-4 and IL-10 alters the sensitivity of cancer cells to chemotherapeutic drug-induced cell death (24). Therefore, the chemoresistant CSCs remaining following standard conventional treatment strategies can effectively accelerate the tumor growth and results in treatment failure and relapse. Therefore, elevated expression levels of ABCG2, IL-4 and Bcl-2 effectively function together and contribute to chemotherapy resistance and tumor relapse. However, the functional interactions between IL-4, Bcl-2 and ABCG2, and their signaling pathways in SP cells require further investigation. Together with others reports, the findings of the present study may assist in further characterizing CSCs for designing effective and novel anticancer drugs.

\section{Acknowledgements}

The authors would like to thank Dr Wanshan Li, Department of Oral and Maxillofacial Surgery, Chongqing Medical University for sharing the FACS protocol by personal communication.

\section{References}

1. Greenlee RT, Hill-Harmon MB, Murray T and Thun M: Cancer statistics, 2001. CA Cancer J. Clin 51: 15-36, 2001.

2. Presnell SC, Petersen B and Heidaran M: Stem cells in adult tissues. Semin Cell Dev Biol 13: 369-376, 2002.

3. Reya T, Morrison SJ, Clarke MF and Weissman LL: Stem cells, cancer, and cancer stem cells. Nature 414: 105-111, 2001.

4. Bonnet D and Dick JE: Human acutemyeloid leukemia is organized as a hierarchy that originates from a primitive hematopoietic cell. Nat Med 3: 730-737, 1997.

5. Collins AT, Berry PA, Hyde C, Stower MJ and Maitland NJ: Prospective identification of tumorigenic prostate cancer stem cells. Cancer Res 65: 10946-10951, 2005.

6. Dontu G, Abdalla WM, Fole JM, et al: In vitro propagation and transcriptional profiling of human mammary stem/progenitor cells. Genes Dev 17: 1253-1270, 2003.

7. Bapat SA, Mali AM, Koppikar CB and Kurrey NK: Stem and progenitor-like cells contribute to the aggressive behavior of human epithelial ovarian cancer, Cancer Res 65:3025-3029, 2005. 
8. Singh SK, Hawkins C, Clarke ID, et al: Identification of human brain tumor initiating cells. Nature 432: 396-401, 2004.

9. Horst D, Kriegl L, Engel J, Kirchner T and Jung A: CD133 expression is an independent prognostic marker for low survival in colorectal cancer. Brit J Cancer 99:1285-1289, 2008.

10. Shapiro HM: Microbial analysis at the single-cell level: Tasks and techniques. J Microbiol Methods 42: 3-16, 2000.

11. Kruger J, Singh K, O'Neill A, Jackson C, Morrison A and O'Brien P: Development of a microfluidic device for fluorescence activated cell sorting. J Micromech Microeng 12: 486-494, 2002

12. Preffer FI, Dombkowski D, Sykes M, Scadden D and Yang YG: Lineage-negative side-population (SP) cells with restricted hematopoietic capacity circulate in normal human adult blood: Immunophenotypic and functional characterization. Stem Cells 20: 417-427, 2002.

13. Dean M, Fojo T and Bates S: Tumor stem cells and drug resistance. Nat Rev Cancer 5: 275-284, 2005.

14. Todaro M,Zerilli M, Ricci-Vitiani L, et al: Autocrine production of interleukin-4 and interleukin-10 is required for survival and growth of thyroid cancer cells. Cancer Res 66: 1491-1499, 2006.

15. Conticello C, Pedini F, Zeuner A, et al: IL-4 protects tumor cells from anti-CD95 and chemotherapeutic agents via up-regulation of antiapoptotic proteins. J Immunol 172: 5467-5477, 2004

16. Qz H, Xz L, Wang K, et al: Isolation and characterization of cancer stem cells from high grade serous ovarian carcinomas. Cell Physiol Biochem 33: 173-184, 2014.
17. Yoo MH and Hatfield DL: The cancer stem cell theory: Is it correct? Mol Cells 26: 514-516, 2008.

18. Hirschmann-Jax C, Foster AE, Wulf GG, et al: A distinct 'side population' of cells with high drug efflux capacity in human tumor cells, Proc Natl Acad USA 101: 14228-14233, 2004.

19. Ricci-Vitiani L, Lombardi DG, Pilozzi E, et al: Identification and expansion of human colon-cancer-initiating cells. Nature 445: 111-115, 2007.

20. Eramo A, Ricci-Vitiani L, Zeuner A, et al: Chemotherapy resistance of glioblastoma stem cells. Cell Death Differ 13: 1238-1241, 2006.

21. Dancescu M, Rubio-Trujillo M, Biron G, Bron D, Delespesse G and Sarfati M: Interleukin 4 protects chronic lymphocytic leukemic B cells from death by apoptosis and upregulates Bcl-2 expression. J Exp Med 176: 1319-1326, 1992.

22. Kieslinger M, Woldman I, Moriggl R, et al: Antiapoptotic activity of Stat5 required during terminal stages of myeloid differentiation. Genes Dev 14: 232-244, 2000.

23. Prokopchuk O, Liu Y, Henne-Bruns D and Kornmann M: Interleukin-4 enhances proliferation of human pancreatic cancer cells: Evidence for autocrine and paracrine actions. Br J Cancer 92: 921- 928, 2005.

24. Todaro M, Perez AM, Di BA, et al: Colon Cancer Stem Cells Dictate Tumor Growth and Resist Cell Death by Production of Interleukin-4. Cell Stem Cell 1: 389-402, 2007. 